\title{
Ray tracing based on the Wigner representation of optical fields
}

\author{
P. Pellat-Finet and E. Fogret \\ 1. Laboratoire de mathématiques de Bretagne Atlantique, UMR CNRS 6205, Université de Bretagne Sud \\ B. P. 92116, 56321 Lorient cedex, France \\ (*) E-mail: pierre.pellat-finet@univ-ubs.fr, eric.fogret@univ-ubs.fr
}

Received: 01/12/2017

Accepted: 01/12/2017

DOI: 10.7149/OPA.51.1.49025

\begin{abstract}
:
An optical ray is defined by its distance to the optical axis of an object, an image, or a refracting surface, and an angle giving its direction of propagation, that is, by a 2-dimensional space vector and a 2dimensional angular frequency. Since we use spherical segments, the angular frequency is related to the angle between the ray and the normal to the spherical segment at the incident point. The Wigner representation of optical fields is a 4-dimensional space-frequency function, defined after choosing appropriate scaled space and frequency variables that are linked to "physical" space variables and angular frequencies. The effect of diffraction on the Wigner distribution of an optical field is deduced from a scalar theory of diffraction and provides a link between scaled space and frequency variables on an emitter and on a receiver at a given distance. By changing scaled variables back into physical ones, we obtain a ray tracing method which is based on a scalar theory of diffraction. An explicit example and an application to optical resonators illustrate the method.
\end{abstract}

Key words: Diffraction, Fractional Fourier optics, Optical resonators, Ray tracing, Wigner distribution

\section{REFERENCES AND LINKS / REFERENCIAS Y ENLACES}

[1] M. A. Alonso, "Wigner functions in optics: describing beams as ray bundles and pulses as particle ensembles", Advances in Optics and Photonics 3, 272-365 (2011).

[2] A. Walther, "Radiometry and Coherence", J. Opt. Soc. Am. 58, 1256-1259 (1968).

[3] M. J. Bastiaans, "Wigner distribution function and its application to first-order optics", J. Opt. Soc. Am. 69, 1710-1716 (1979).

[4] T. Cuypers, T. Haber, P. Bekaert, S. B. Oh, R. Raskar, "Reflectance Model for Diffraction", ACM Transactions on Graphics 28, Art. 106 (2009).

[5] T. Cuypers, R. Horstmeyer, P. Bekaert, R. Raskar, "Validity of Wigner distribution function for ray-based imaging", IEEE International Conference on Computational Photography (ICCP), Section 4 (2011).

[6] B. M. Mout, M. Wick, F. Bociort, H. P. Urbach, "A Wigner-based-ray-tracing method for imaging simulations", in Optical Systems Design: Computational Optics, Daniel G. Smith, Frank Wyrowski, Andreas Erdmann Eds., Proc. SPIE 9630, 96300Z-1 - 96300Z-11 (2015).

[7] J. W. Goodman, Introduction to Fourier optics, 3rd Ed., Englewood, Robert \& Compagny (2005).

[8] P. Pellat-Finet, Optique de Fourier, théorie métaxiale et fractionnaire, Paris, Springer (2009).

[9] V. Namias, "The fractional order Fourier transform and its application to quantum mechanics", J. Inst. Maths Applics 25, 241-265 (1980).

[10] H. M. Ozaktas, Z. Zalzevsky, M. A. Kutay, The Fractional Fourier Transform, with Applications in Optics and Signal Processing, Chichester, John Wiley \& Sons (2001).

[11] P. Pellat-Finet, E. Fogret, "Complex order fractional Fourier transforms and their use in diffraction theory", Opt. Comm. 258, 103-113 (2006). 
[12] P. Pellat-Finet, E. Fogret, "A fractional Fourier Transform Theory of Optical Resonators", in Peter S. Emersone Ed. Progress in Optical Fibers, New York, Nova Science Publisher, 299-351 (2011).

[13] P. Pellat-Finet, P.-E. Durand, E. Fogret, "Spherical angular spectrum and the fractional order Fourier transform", Opt. Lett. 31, 3429-3431 (2006).

\section{Introduction}

A lot of works have been devoted to Wigner distributions in many areas, such as Quantum Mechanics or Signal Processing [1]. In Optics, since the work of Walther [2] and Bastiaans [3], Wigner distributions have been used for representing optical fields, for dealing with radiometry and coherence theory [2], with applications to tomography [1], or recently for simulating wave effects in graphics [4].

We introduce here a new method of ray tracing based on the Wigner representation of optical fields and on its propagation by diffraction from an emitter to a receiver. The new method is different from those that have been recently proposed by several authors and that are also based on Wigner distributions [5,6].

Since we use spherical segments in place of reference planes, the angular variable associated with a ray is related to the angle between the ray and the normal to the reference sphere at the incident point. Doing so is different from what is done in ray tracing methods that are commonly used in geometrical optics. It leads to a more direct use of Snell law and allow us to easily include ray tracing for skew rays as we will see.

\section{Geometrical parameters}

\section{2. a. Defining spatial and angular variables}

We begin by adapting to spherical segments a light ray representation that is currently used in geometrical optics and according to which a light ray emerging from a transverse plane is represented by a transverse vector and an angle providing the propagation direction of the ray.

In the framework of a scalar theory of diffraction, light propagation is expressed by Fresnel-type integrals that include quadratic phase factors $[7,8]$. These factors result from phase differences that exist between field amplitudes taken on two spherical segments, or on a plane and a spherical segment; they can be handled by considering emitters and receivers that are spherical segments [8]. The interest of dealing with spherical objects and images, not only plane ones, is reinforced by considering that refracting surfaces as well as mirrors generally are spherical segments too. (Plane surfaces are included in our approach: their radii of curvature are infinite.)

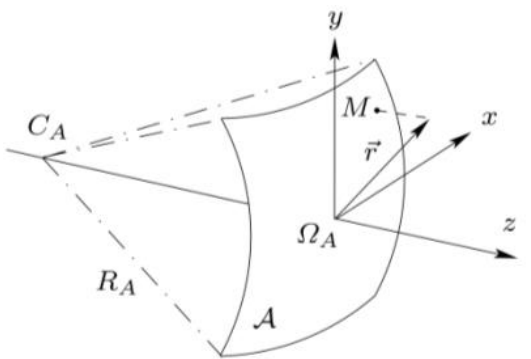

Fig. 1: Coordinates of a point $M$ on the spherical segment $\mathcal{A}$.

Let $\mathcal{A}$ be a spherical segment: its radius of curvature is $R_{A}=\overline{\Omega_{A} C_{A}}$, where $\Omega_{A}$ is the vertex of $\mathcal{A}$ and $C_{A}$ its center of curvature (see Fig. 1). We choose Cartesian coordinates $(x, y)$ in the plane $\mathcal{P}$ tangent to $\mathcal{A}$ at $\Omega_{A}$. Let $M$ be a point on $\mathcal{A}$ and let $m$ be its projection on $\mathcal{P}$. The coordinates of $m$ in $\mathcal{P}$ are $x_{m}$ and $y_{m}$. We write $\overrightarrow{\Omega_{A} m}=\vec{r}=\left(x_{m}, y_{m}\right)$. Coordinates $x_{m}$ and $y_{m}$ can be used to localize $M$ on the spherical segment $\mathcal{A}$ : they are the coordinates of $M$ on the spherical segment $\mathcal{A}$. In the following, the index $m$ will be dropped.

Let $z$ be the axis along light propagation direction and let $\overrightarrow{e_{x}}, \overrightarrow{e_{y}}$ and $\overrightarrow{e_{z}}$ be unit vectors along axes $x, y$ and $z$, and forming a direct basis $\left(\overrightarrow{e_{x}}=\overrightarrow{e_{y}} \times \overrightarrow{e_{z}}\right)$. Let $M$ be a point on $\mathcal{A}$ and let $\overrightarrow{e_{n}}$ be the vector normal to $\mathcal{A}$ at $M$ and such that the Euclidean scalar product $\overrightarrow{e_{n}} \cdot \overrightarrow{e_{x}}$ is positive. We introduce the following unit vectors

$$
\overrightarrow{e_{\xi}}=\frac{\overrightarrow{e_{y}} \times \overrightarrow{e_{n}}}{\left\|\overrightarrow{e_{y}} \times \overrightarrow{e_{n}}\right\|},
$$




$$
\overrightarrow{e_{\eta}}=\frac{\overrightarrow{e_{n}} \times \overrightarrow{e_{x}}}{\left\|\overrightarrow{e_{n}} \times \overrightarrow{e_{x}}\right\|}
$$

that form a direct basis with $\overrightarrow{e_{n}}$ (that is: $\overrightarrow{e_{n}}=\overrightarrow{e_{\xi}} \times \overrightarrow{e_{\eta}}$ ). Vectors $\overrightarrow{e_{\xi}}$ and $\overrightarrow{e_{\eta}}$ both lay in the plane tangent to $\mathcal{A}$ at $M$ (see Fig. 2).
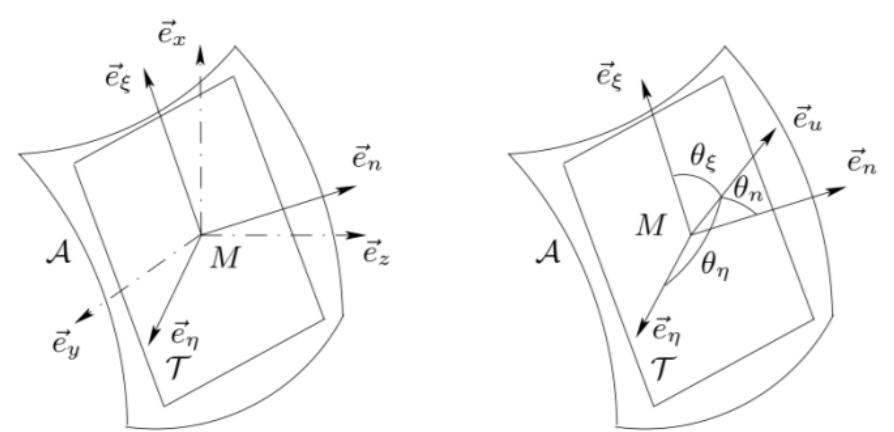

Fig. 2: Definition of director cosines for a given ray passing by $M$ and propagating along $\overrightarrow{e_{u}}$.

We consider a light ray passing by $M$ and let $\overrightarrow{e_{u}}$ be the unit vector along the ray (in the direction of propagation). We denote by $\theta_{\xi}, \theta_{\eta}$ and $\theta_{n}$ the angles of $\overrightarrow{e_{u}}$ with $\overrightarrow{e_{\xi}}, \overrightarrow{e_{\eta}}$ and $\overrightarrow{e_{n}}$ respectively (taken from $\overrightarrow{e_{k}}$, $k=\xi, \eta, n$, to $\left.\overrightarrow{e_{u}}\right)$. The director cosines of $\overrightarrow{e_{u}}$ with $\overrightarrow{e_{\xi}}, \overrightarrow{e_{\eta}}$ and $\overrightarrow{e_{n}}$ are

$$
\begin{gathered}
\xi=\cos \theta_{\xi}, \\
\eta=\cos \theta_{\eta}, \\
\zeta=\cos \theta_{n} .
\end{gathered}
$$

Indeed, since $\overrightarrow{e_{u}}$ is unitary, we have $\xi^{2}+\eta^{2}+\zeta^{2}=1$, so that $\overrightarrow{e_{u}}$ is perfectly defined by $\xi$ and $\eta$. We call angular frequency vector the 2-dimensional vector $\vec{\Phi}$ defined by

$$
\vec{\Phi}=(\xi, \eta) .
$$

The previous light ray, that intercepts $\mathcal{A}$ at $M$, is perfectly defined by the pair $(\vec{r}, \vec{\Phi})$ so that we shall say "the ray $(\vec{r}, \vec{\Phi}) "$.

The same method can be applied to every ray that intercepts $\mathcal{A}$ at an arbitrary point. Moreover $\mathcal{A}$ can be an arbitrary spherical segment.

Finally, the problem of ray tracing can be expressed in the following form: given two spherical segments $\mathcal{A}$ and $\mathcal{B}$ and given a ray $(\vec{r}, \vec{\Phi})$ on $\mathcal{A}$, find the corresponding ray $\left(\overrightarrow{r^{\prime}}, \overrightarrow{\Phi^{\prime}}\right)$ on $\mathcal{B}$ (see Fig. 3).

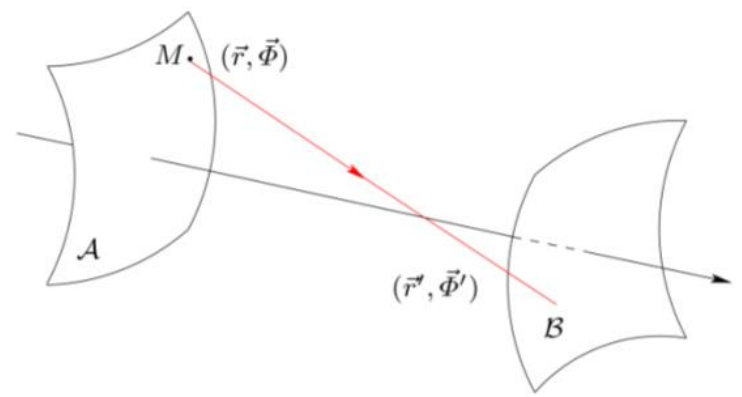

Fig. 3: Ray tracing: Given a ray $(\vec{r}, \vec{\Phi})$ on $\mathcal{A}$, find the corresponding ray $\left(\overrightarrow{r^{\prime}}, \overrightarrow{\Phi^{\prime}}\right)$ on $\mathcal{B}$.

We point out that $\vec{\Phi}$ provides the angle between the ray and the normal of $\mathcal{A}$ at point $M$, and $\overrightarrow{\Phi^{\prime}}$ the angle between the ray and the normal of $\mathcal{B}$ at the incident point. This is why we generally have $\vec{\Phi} \neq \overrightarrow{\Phi^{\prime}}$. 


\section{2. b. Snell law}

Ray tracing also includes rays passing through refracting surfaces. In vectorial form, Snell law is (see Fig. 4)

$$
n \vec{\Phi}=n^{\prime} \overrightarrow{\Phi^{\prime}}
$$

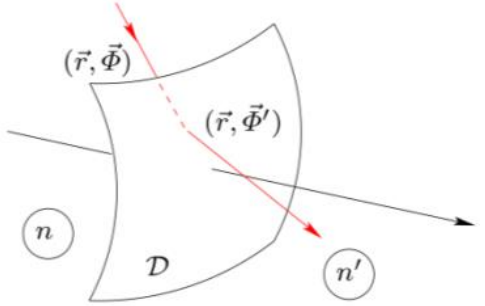

Fig. 4: Illustrating Snell law. The refracting surface $\mathcal{D}$ separates two homogeneous media whose respective refractive indices are $n$ and $n^{\prime}$.

If $0 \leq \theta_{n} \leq \pi / 2$ (see Fig. 2), we have $\|\vec{\Phi}\|=\sin \theta_{n}$; if $-\pi / 2 \leq \theta_{n} \leq 0$, then $\|\vec{\Phi}\|=-\sin \theta_{n}$ : Eq. (7) includes the sinus law of refraction. When expressed in vectorial form, Snell law applies to skew rays: The incidence plane is not necessarily a meridian plane for the refracting surface $\mathcal{D}$.

\section{Field transfer by diffraction and the fractional order Fourier transform}

\section{3. a. Transfer of the field amplitude (spatial variables)}

According to a scalar theory of diffraction, given the wavelength $\lambda$, the field transfer from a spherical emitter $\mathcal{A}$ (radius $R_{A}$, spatial variable $\vec{r}$ ) to a spherical receiver $\mathcal{B}$ (radius $R_{B}$, spatial variable $\vec{s}$ ) at a distance $D$ (see Fig. 5) is expressed by [8]

$$
U_{B}(\vec{s})=\frac{i}{\lambda D} \exp \left[-\frac{i \pi}{\lambda}\left(\frac{1}{R_{B}}+\frac{1}{D}\right) s^{2}\right] \int_{\mathbb{R}^{2}} \exp \left[-\frac{i \pi}{\lambda}\left(\frac{1}{D}-\frac{1}{R_{A}}\right) r^{2}\right] \exp \left(\frac{2 i \pi}{\lambda D} \vec{r} \cdot \vec{s}\right) U_{A}(\vec{r}) d \vec{r},
$$

where $U_{A}$ is the complex (electric) field amplitude on $\mathcal{A}$, and $U_{B}$ on $\mathcal{B}$, and where $\mathrm{d} \vec{r}=\mathrm{d} x \mathrm{~d} y$, if $\vec{r}=(x, y)$. A constant multiplicative phase factor, of the form $\exp (-2 i \pi D / \lambda)$, has been omitted in Eq. (8).

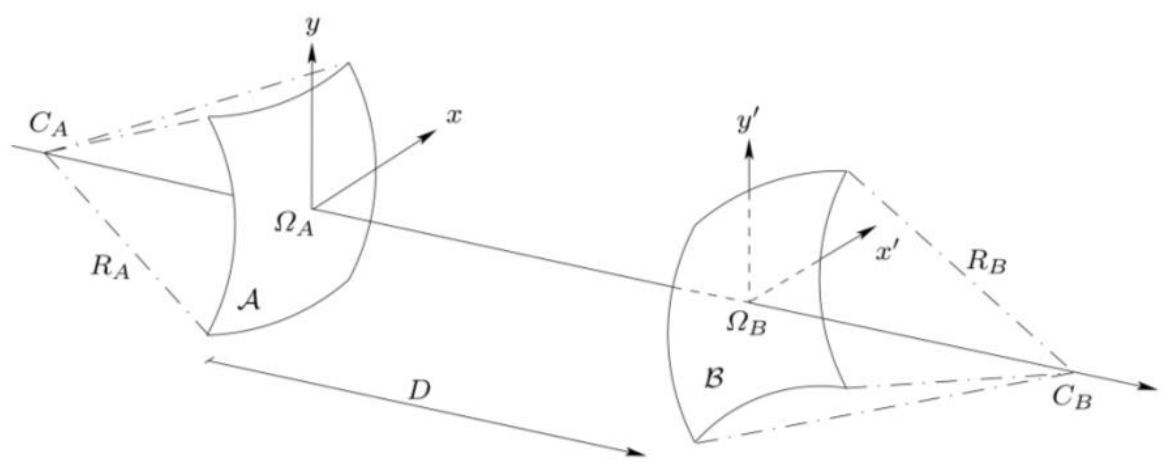

Fig. 5: Diffraction from emitter $\mathcal{A}$ to receiver $\mathcal{B}$.

On the other hand, the 2-dimensional fractional Fourier transform of order $\alpha$ of function $f$ is defined by [810]

$$
\mathcal{F}_{\alpha}[f](\vec{\sigma})=\frac{i \mathrm{e}^{-i \alpha}}{\sin \alpha} \exp \left(-i \pi \sigma^{2} \cot \alpha\right) \int_{\mathbb{R}^{2}} \exp \left(-i \pi \rho^{2} \cot \alpha\right) \exp \left(\frac{2 i \pi \vec{\sigma} \cdot \vec{\rho}}{\sin \alpha}\right) f(\vec{\rho}) d \vec{\rho},
$$

where $\vec{\rho}$ and $\vec{\sigma}$ are 2-dimensional real vectors. The order $\alpha$ may be a complex number. The usual (or standard) Fourier transform is $\mathcal{F}_{\pi / 2}$, and for every function $f$, we have $\mathcal{F}_{0}[f]=f$.

To express Eq. (8) in the form of a fractional order Fourier transform, we process as follows. Let $K$ be such that

$$
K=\left(1-\frac{D}{R_{A}}\right)\left(1+\frac{D}{R_{B}}\right)
$$


For the sake of simplicity, we assume $0 \leq K \leq 1$, and we define $\alpha$ (a real number) by

$$
\cos ^{2} \alpha=K, \quad-\pi \leq \alpha \leq \pi, \quad \alpha D \geq 0 .
$$

An equivalent definition, that is useful in some derivations, is

$$
\cot ^{2} \alpha=\frac{\left(D+R_{B}\right)\left(R_{A}-D\right)}{D\left(D-R_{A}+R_{B}\right)}, \quad-\pi \leq \alpha \leq \pi, \quad \alpha D \geq 0 .
$$

We then define auxiliary parameters $\varepsilon_{A}$ and $\varepsilon_{B}$ by

$$
\begin{gathered}
\varepsilon_{A}=\frac{D}{R_{A}-D} \cot \alpha, \quad \varepsilon_{A} R_{A}>0, \\
\varepsilon_{B}=\frac{D}{D+R_{B}} \cot \alpha .
\end{gathered}
$$

(Then, necessarily $\varepsilon_{B} R_{B}>0$.)

Eventually we choose scaled spatial variables $\vec{\rho}$ on $\mathcal{A}$ and $\vec{\sigma}$ on $\mathcal{B}$ according to

$$
\vec{\rho}=\frac{\vec{r}}{\sqrt{\lambda \varepsilon_{A} R_{A}}}, \quad \vec{\sigma}=\frac{\vec{s}}{\sqrt{\lambda \varepsilon_{B} R_{B}}} .
$$

We use scaled field amplitudes $V_{A}$ on $\mathcal{A}$ and $V_{B}$ on $\mathcal{B}$ such that

$$
V_{A}(\vec{\rho})=\sqrt{\frac{\varepsilon_{A} R_{A}}{\lambda}} U_{A}\left(\sqrt{\lambda \varepsilon_{A} R_{A}} \vec{\rho}\right), \quad V_{B}(\vec{\sigma})=\sqrt{\frac{\varepsilon_{B} R_{B}}{\lambda}} U_{B}\left(\sqrt{\lambda \varepsilon_{B} R_{B}} \vec{\sigma}\right) .
$$

Then, Eq. (8) becomes

$$
V_{B}(\vec{\sigma})=\mathrm{e}^{i \alpha} \mathcal{F}_{\alpha}\left[V_{A}\right](\vec{\sigma}) \text {. }
$$

Eq. (17) expresses a Fresnel diffraction phenomenon in the form of a fractional Fourier transform whose order depends on the propagation distance and the radii of the emitter and the receiver. Fraunhofer diffraction is obtained for $\alpha=\pi / 2$ (Fourier transform).

If $K>1$, or $K<0$, the order $\alpha$ becomes a complex number: it also correponds to some diffraction phenomenon, depending on propagation distance and radii of curvature $[8,11,12]$.

In order not to repeat definitions of parameters every time, we will write

$$
\left(R_{A}, R_{B}, D, \vec{r}, \vec{s}, U_{A}, U_{B}, \lambda\right) \leftrightarrow\left(\alpha, \varepsilon_{A}, \varepsilon_{B}, \vec{\rho}, \vec{\sigma}, V_{A}, V_{B}\right),
$$

to indicate the correspondence between physical parameters and variables (on the left side) and fractional parameters, scaled variables and scaled field amplitudes (on the right).

\section{3. b. Transfer of the spherical angular spectrum (frequency variables)}

Let $\widehat{U_{A}}$ be the standard Fourier transform of $U_{A}$

$$
\widehat{U_{A}}(\vec{F})=\int_{\mathbb{R}^{2}} \exp (2 i \pi \vec{r} \cdot \vec{F}) U_{A}(\vec{r}) \mathrm{d} \vec{r}
$$

where $\vec{F}$ is a spatial frequency, that is, the conjugate variable of the spatial variable $\vec{r}$.

Spatial frequencies and angular frequency vectors are linked according to

$$
\vec{\Phi}=\lambda \vec{F} .
$$

The spherical angular spectrum of the field amplitude $U_{A}$ on $\mathcal{A}$ is [13]

$$
S_{A}(\vec{\Phi})=\frac{1}{\lambda^{2}} \widehat{U_{A}}\left(\frac{\vec{\Phi}}{\lambda}\right)
$$


The propagation of the spherical angular spectrum from the emitter $\mathcal{A}$ to the receiver $\mathcal{B}$ at a distance $D$ is expressed by [13]

$$
\begin{aligned}
S_{B}\left(\overrightarrow{\Phi^{\prime}}\right)=\frac{i R_{A} R_{B}}{\lambda\left(D-R_{A}+R_{B}\right)} \exp \left[-\frac{i \pi R_{B}\left(R_{A}-D\right)}{\lambda\left(D-R_{A}+R_{B}\right)} \Phi^{\prime 2}\right] \\
\qquad \int_{\mathbb{R}^{2}} \exp \left[-\frac{i \pi R_{A}\left(D+R_{B}\right)}{\lambda\left(D-R_{A}+R_{B}\right)} \Phi^{2}\right] \exp \left(\frac{2 i \pi R_{A} R_{B}}{\lambda\left(D-R_{A}+R_{B}\right)} \vec{\Phi} \cdot \overrightarrow{\Phi^{\prime}}\right) S_{A}(\vec{\Phi}) \mathrm{d} \vec{\Phi}
\end{aligned}
$$

Eq. (22) is no more than Eq. (8) where field amplitudes $U_{A}$ and $U_{B}$ are replaced by $S_{A}$ and $S_{B}$, vectors $\vec{r}$ and $\overrightarrow{r^{\prime}}$ by $\vec{\Phi}$ and $\overrightarrow{\Phi^{\prime}}$, and where $D, R_{A}$ and $R_{B}$ are changed according to

$$
\begin{aligned}
& D \mapsto \frac{D-R_{A}+R_{B}}{R_{A} R_{B}}, \\
& R_{A} \mapsto \frac{D-R_{A}+R_{B}}{D R_{A}}, \\
& R_{B} \mapsto \frac{D-R_{A}+R_{B}}{D R_{B}} .
\end{aligned}
$$

If we choose $\alpha, \varepsilon_{A}$ and $\varepsilon_{B}$ as in Eqs. (11), (13) and (14), and define scaled angular variables by

$$
\vec{\phi}=\sqrt{\frac{\varepsilon_{A} R_{A}}{\lambda}} \vec{\Phi}, \quad \overrightarrow{\phi^{\prime}}=\sqrt{\frac{\varepsilon_{B} R_{B}}{\lambda}} \overrightarrow{\Phi^{\prime}},
$$

and scaled angular spectra by

$$
T_{A}(\vec{\phi})=\sqrt{\frac{\lambda}{\varepsilon_{A} R_{A}}} S_{A}\left(\sqrt{\frac{\lambda}{\varepsilon_{A} R_{A}}} \vec{\phi}\right), \quad T_{B}\left(\overrightarrow{\phi^{\prime}}\right)=\sqrt{\frac{\lambda}{\varepsilon_{B} R_{B}}} S_{B}\left(\sqrt{\frac{\lambda}{\varepsilon_{B} R_{B}}} \overrightarrow{\phi^{\prime}}\right),
$$

then Eq. (22) becomes

$$
T_{B}=\mathrm{e}^{i \alpha} \mathcal{F}_{\alpha}\left[T_{A}\right] .
$$

Eq. (28) is no more than Eq. (17), but holds for propagation of the scaled spherical angular spectrum.

We have $T_{A}=\widehat{V_{A}}$, so that Eq. (28) can be written

$$
\widehat{V_{B}}=\mathrm{e}^{i \alpha} \mathcal{F}_{\alpha}\left[\widehat{V_{A}}\right] \text {. }
$$

According to Eqs. (17) and (29) we obtain the following commutative diagram

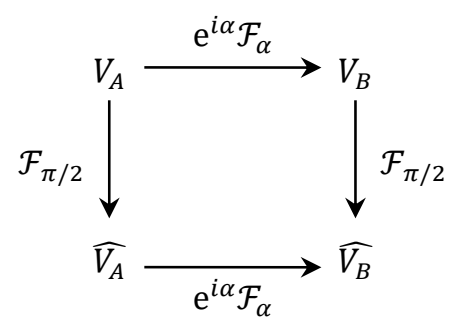

The fact that propagations of both the scaled field amplitude and the scaled spherical angular spectrum are expressed in the same way when using a fractional order Fourier transform will be a key point in studying the effect of diffraction on the Wigner representation of an optical field, since it will allow us to introduce rotations in the scaled Wigner space.

\section{Fresnel diffraction and Wigner distribution}

\section{4. a. Wigner representation of optical fields}


The Wigner distribution of the optical field on a spherical segment $\mathcal{A}$ is defined by

$$
W_{A}(\vec{\rho}, \vec{\phi})=\int_{\mathbb{R}^{2}} V_{A}\left(\vec{\rho}+\frac{\vec{\sigma}}{2}\right) \overline{V_{A}\left(\vec{\rho}-\frac{\vec{\sigma}}{2}\right)} \mathrm{e}^{2 i \pi \vec{\sigma} \cdot \vec{\phi}} \mathrm{d} \vec{\sigma},
$$

where $V_{A}$ is the scaled field amplitude on $\mathcal{A}$ and $\overline{V_{A}}$ its complex conjugate. Indeed $W_{A}$ should be called the scaled Wigner function (or distribution) associated with the field amplitude on $\mathcal{A}$. It can be proved that

$$
W_{A}(\vec{\rho}, \vec{\phi})=\int_{\mathbb{R}^{2}} \widehat{V_{A}}\left(\vec{\phi}+\frac{\vec{\theta}}{2}\right) \overline{\widehat{V_{A}}\left(\vec{\phi}-\frac{\vec{\theta}}{2}\right)} \mathrm{e}^{2 i \pi \vec{\rho} \cdot \vec{\theta}} \mathrm{d} \vec{\theta}
$$

The Wigner distribution $W_{A}$ is defined on a 4-dimensional space (variables $\vec{\rho}$ and $\vec{\phi}$ ) called the scaled Wigner space.

\section{4. b. Effect of diffraction on the Wigner distribution of an optical field}

Let $\alpha$ be the order of the fractional Fourier transform associated with the field transfer from a spherical emitter $\mathcal{A}$ to spherical receiver $\mathcal{B}$ at a distance $D$. For real $\alpha$, the Wigner distributions of the fields on $\mathcal{A}$ and $\mathcal{B}$ are linked as follows

$$
W_{B}(\vec{\rho}, \vec{\phi})=W_{A}(\vec{\rho} \cos \alpha-\vec{\phi} \sin \alpha, \vec{\rho} \sin \alpha+\vec{\phi} \cos \alpha) .
$$

According to Eq. (33), the effect of Fresnel diffraction is a rotation of the Wigner distribution associated with the scaled field amplitude. The rotation operates in the 4-dimensional phase space $\vec{\rho}-\vec{\phi}$. The value of $W_{B}$ at point $(\vec{\rho}, \vec{\phi})$ is equal to the value of function $W_{A}$ at point $(\vec{\rho} \cos \alpha-\vec{\phi} \sin \alpha, \vec{\rho} \sin \alpha+\vec{\phi} \cos \alpha)$.

The effect of diffraction on the Wigner distribution can be expressed by a matrix. We use $\vec{\rho}=\left(\rho_{x}, \rho_{y}\right)$ and $\vec{\phi}=\left(\phi_{x}, \phi_{y}\right)$, so that

$$
\left(\begin{array}{l}
\rho_{x_{B}} \\
\phi_{x_{B}} \\
\rho_{y_{B}} \\
\phi_{y_{B}}
\end{array}\right)=\left(\begin{array}{cccc}
\cos \alpha & \sin \alpha & 0 & 0 \\
-\sin \alpha & \cos \alpha & 0 & 0 \\
0 & 0 & \cos \alpha & \sin \alpha \\
0 & 0 & -\sin \alpha & \cos \alpha
\end{array}\right)\left(\begin{array}{c}
\rho_{x_{A}} \\
\phi_{x_{A}} \\
\rho_{y_{A}} \\
\phi_{y_{A}}
\end{array}\right) .
$$

The transformation in Eq. (34) can split into two 2-dimensional rotations, whose angles are equal to $-\alpha$. For example

$$
\left(\begin{array}{l}
\rho_{x_{B}} \\
\phi_{x_{B}}
\end{array}\right)=\left(\begin{array}{cc}
\cos \alpha & \sin \alpha \\
-\sin \alpha & \cos \alpha
\end{array}\right)\left(\begin{array}{l}
\rho_{x_{A}} \\
\phi_{x_{A}}
\end{array}\right)
$$

Fig. 6 graphically shows how scaled parameters of the ray on $\mathcal{B}$ can be deduced from scaled parameters of the ray on $\mathcal{A}$. The graphic is drawn in a 2-dimensional subspace of the 4-dimensional Wigner space.

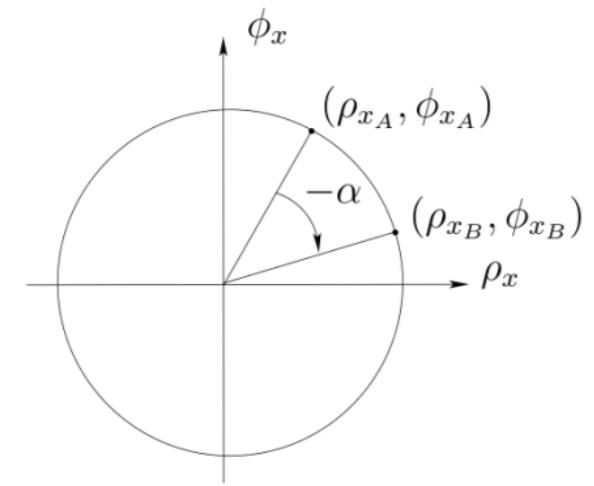

Fig. 6: Scaled parameters of the ray on $\mathcal{B}$ are deduced from scaled parameters of the ray on $\mathcal{A}$ in a rotation of angle $-\alpha$. This basic property will be used for ray tracing.

It happens that $\alpha$ is a complex number of the form $\alpha=\mathrm{i} \beta$. Then the matrix rotation in Eq. (35) becomes the matrix of a hyperbolic rotation, that is 


$$
\begin{gathered}
\begin{array}{c}
\text { ÓPTICA PURA Y APLICADA } \\
\text { www.sedoptica.es }
\end{array} \\
\left(\begin{array}{c}
\rho_{x_{B}} \\
\phi_{x_{B}}
\end{array}\right)=\left(\begin{array}{ll}
\cosh \beta & \sinh \beta \\
\sinh \beta & \cosh \beta
\end{array}\right)\left(\begin{array}{l}
\rho_{x_{A}} \\
\phi_{x_{A}}
\end{array}\right) .
\end{gathered}
$$

Then circles as in Fig. 6 are replaced by hyperbolae.

\section{Ray tracing}

\section{5. a. The method}

To illustrate our method, we consider an emitter $\mathcal{A}$ (radius $R_{A}$, spatial variable $\overrightarrow{r_{A}}$ ), a refracting surface $\mathcal{D}$ (radius $R_{D}$, spatial variable $\overrightarrow{r_{D}}$ ) at a distance $D$, and a receiver $\mathcal{B}$ (radius $R_{B}$, spatial variable $\overrightarrow{r_{B}}$ ) at a distance $D^{\prime}$ from $\mathcal{D}$ (see Fig. 7).

The field transfer from $\mathcal{A}$ to $\mathcal{D}$ corresponds to

$$
\left(R_{A}, R_{D}, D, \overrightarrow{r_{A}}, \overrightarrow{r_{D}}, U_{A}, U_{D}, \lambda\right) \leftrightarrow\left(\alpha, \varepsilon_{A}, \varepsilon_{D}, \overrightarrow{\rho_{A}}, \overrightarrow{\rho_{D}}, V_{A}, V_{D}\right),
$$

and the field transfer from $\mathcal{D}$ to $\mathcal{B}$ corresponds to

$$
\left(R_{D}, R_{B}, D^{\prime}, \overrightarrow{r_{D}}, \overrightarrow{r_{B}}, U_{D}, U_{B}, \lambda^{\prime}\right) \leadsto\left(\alpha^{\prime}, \varepsilon_{D}^{\prime}, \varepsilon_{B}^{\prime}, \overrightarrow{\rho_{D}^{\prime}}, \overrightarrow{\rho_{B}^{\prime}}, V_{D}^{\prime}, V_{B}^{\prime}\right) .
$$

The basic idea is using Eq. (34) to deduce the ray on $\mathcal{D}$ corresponding to a given ray on $\mathcal{A}$.

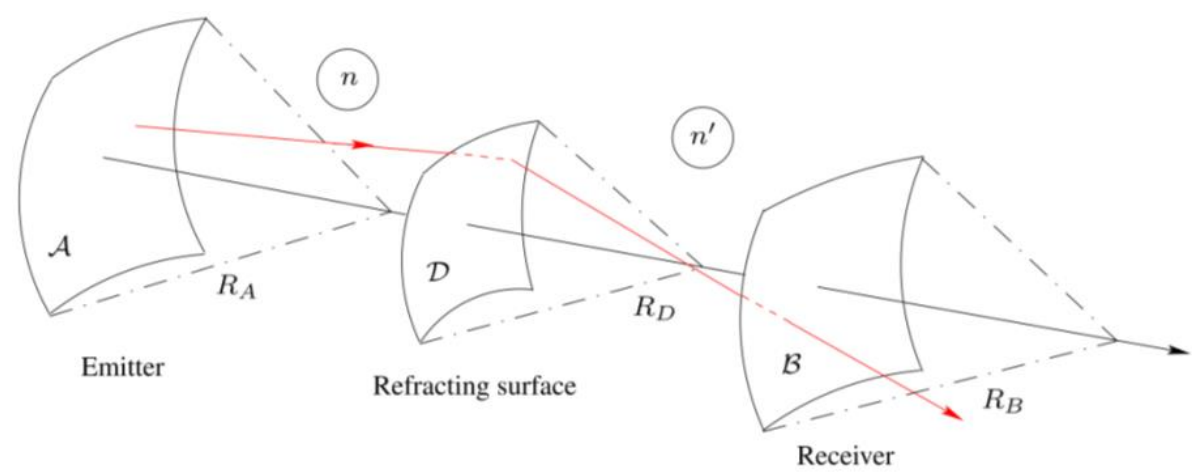

Fig. 7: Ray tracing from $\mathcal{A}$ to $\mathcal{B}$

Let $\left(\overrightarrow{r_{A}}, \overrightarrow{\Phi_{A}}\right)=\left(x_{A}, y_{A}, \Phi_{x_{A}}, \Phi_{y_{A}}\right)$ be a ray on $\mathcal{A}$. The corresponding ray, incident on $\mathcal{D}$, is $\left(\overrightarrow{r_{D}}, \overrightarrow{\Phi_{D}}\right)$; it is obtained according to the following steps.

1. Calculate $\alpha$, according to Eq. (10);

2. Calculate $\varepsilon_{A}$ and deduce scaled variables $\overrightarrow{\rho_{A}}=\left(\rho_{x_{A}}, \rho_{y_{A}}\right)$ and $\overrightarrow{\phi_{A}}=\left(\phi_{x_{A}}, \phi_{y_{A}}\right)$ from $\overrightarrow{r_{A}}$ and $\overrightarrow{\Phi_{A}}$, according to Eqs. (15) and (26) (after adapting notations);

3. Apply Eq. (34) and obtain scaled variables on $\mathcal{D}: \overrightarrow{\rho_{D}}=\left(\rho_{x_{D}}, \rho_{y_{D}}\right)$ and $\overrightarrow{\phi_{D}}=\left(\phi_{x_{D}}, \phi_{y_{D}}\right)$;

4. Calculate $\varepsilon_{D}$ and deduce $\left(\overrightarrow{r_{D}}, \overrightarrow{\Phi_{D}}\right)$ from $\overrightarrow{\rho_{D}}$ and $\overrightarrow{\phi_{D}}$, according to Eqs. (15) and (26).

Then we apply Snell law: $n \overrightarrow{\Phi_{D}}=n^{\prime} \overrightarrow{\Phi_{D}^{\prime}}$, so that the ray emerging from $\mathcal{D}$ should be $\left(\overrightarrow{r_{D}^{\prime}}, \overrightarrow{\Phi_{D}^{\prime}}\right)$ such that

$$
\left(\overrightarrow{r_{D}^{\prime}}, \overrightarrow{\Phi_{D}^{\prime}}\right)=\left(\overrightarrow{r_{D}}, \frac{n}{n^{\prime}} \overrightarrow{\Phi_{D}}\right) \text {. }
$$

Finally, the ray on $\mathcal{B}$ is obtained by applying the previous steps $1-4$ to $\left(\overrightarrow{r_{D}^{\prime}}, \overrightarrow{\Phi_{D}^{\prime}}\right)$, replacing $\alpha$ by $\alpha^{\prime}, \varepsilon_{A}$ by $\varepsilon_{D}^{\prime}$, and so on, according to Eqs. (37) and (38).

\section{5. b. A numerical example}

We consider an emitter $\mathcal{A}$ whose curvature radius is $R_{A}=20 \mathrm{~cm}$, and a receiver $\mathcal{B}$ whose radius is $R_{B}=$ $-20 \mathrm{~cm}$. The distance from $\mathcal{A}$ to $\mathcal{B}$ is $D=10 \mathrm{~cm}$. The wavelength is $\lambda=0.5 \mu \mathrm{m}$. 
Table 1: Numerical example

\begin{tabular}{crl}
\hline Parameter & Value & Deduced from \\
\hline$\alpha$ & $\pi / 3$ & Eqs. (10) and (11) \\
$\varepsilon_{A}$ & 0.57735 & Eq. (13) \\
$\overrightarrow{\varepsilon_{B}}$ & -0.57735 & Eq. (14) \\
$\overrightarrow{\rho_{A}}$ & $(41.618,0)$ & Eq. (15) \\
$\overrightarrow{\phi_{A}}$ & $(208,0)$ & Eq. (26) \\
$\overrightarrow{\rho_{B}}$ & $(200.94,0)$ & Eq. (34) \\
$\overrightarrow{\phi_{B}}$ & $(104,0)$ & Eq. (34) \\
$\overrightarrow{r_{B}}$ & $(4.82 \mathrm{~cm}, 0)$ & Eq. (15) \\
$\overrightarrow{\Phi_{B}}$ & $(0.21641,0)$ & Eq. (26) \\
\hline
\end{tabular}

The initial ray on $\mathcal{A}$ originates at point $M=\overrightarrow{r_{A}}=(1 \mathrm{~cm}, 0)$ and makes an angle of $30^{\circ}$ with the normal at $M$, so that $\overrightarrow{\Phi_{A}}=(0.5,0)$ (since $\theta_{\xi}=60^{\circ}$ ).

Numerical values of parameters and variables are given in Tab. 1.

We find that the ray is incident on $\mathcal{B}$ at point $P=\overrightarrow{r_{B}}=(4.82 \mathrm{~cm}, 0)$, and since $\left\|\overrightarrow{\Phi_{B}}\right\|=\sin \theta_{n_{B}}=0.21641$, the ray makes an angle of $12.5^{\circ}$ with the normal to $\mathcal{B}$ at $P$.

\section{Application to optical resonators}

We consider an optical resonator made up of two spherical mirrors, say $\mathcal{M}_{1}$ and $\mathcal{M}_{2}$ (see Fig. 8). Let $\alpha$ be the order of the fractional Fourier transform associated with the field transfer from $\mathcal{M}_{1}$ to $\mathcal{M}_{2}$. It can be shown that $\alpha$ is also the order of the fractional Fourier transform associated with the field transfer from $\mathcal{M}_{2}$ to $\mathcal{M}_{1}$ [12]. Then multiple field transfers from a mirror to the other are represented by successive rotations of the Wigner distribution. Successive representative points of a ray in a 2-dimensional subspace of the Wigner space are deduced from each other by a rotation of angle $-\alpha$.

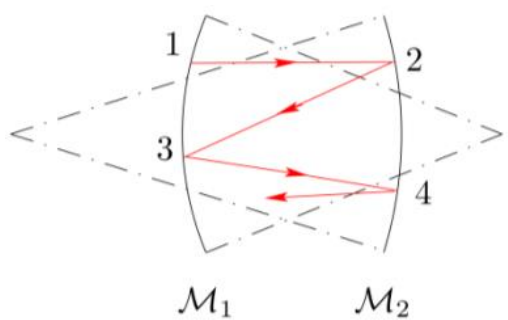

Fig. 8: An optical resonator, and several reflections of a ray.

If $q \alpha=2 \pi(q \in \mathbb{N})$, the representative point of a ray after $q$ reflections is the initial point once more. The ray is said to be "reentrant". We provide an example. Let $\mathcal{M}_{1}$ (radius $R_{1}$ ) and $\mathcal{M}_{2}$ (radius $R_{2}$ ) be such that $R_{1}=2 D=-R_{2}$, where $D$ is the distance between $\mathcal{M}_{1}$ and $\mathcal{M}_{2}$ (see Fig. 9). Then $\cos ^{2} \alpha=1 / 4$ and $\alpha=\pi / 3$, so that $q=6$. The ray superposes with itself after 6 reflections.

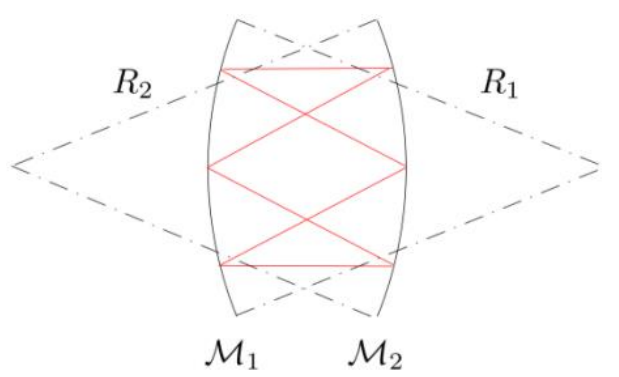

Fig. 9: Reentrant ray in an optical resonator. 


\section{Conclusion}

The proposed method for ray tracing, based on the Wigner representation of optical fields, applies for meridian rays as well as for skew rays. Since angles are taken between a ray and the normal to a reference sphere at incident point, application of Snell law, in its vectorial form, is straightforward, for meridian or skew rays. 